\title{
On the Convergence Rate of Distributed Gradient Methods for Finite-Sum Optimization under Communication Delays
}

\author{
Thinh T. Doan \\ University of Illinois at \\ Urbana-Champaign \\ Urbana, IL, USA \\ ttdoan2@illinois.edu
}

\author{
Extended Abstract ${ }^{*}$ \\ Carolyn L. Beck \\ University of Illinois at \\ Urbana-Champaign \\ Urbana, IL, USA \\ beck3@illinois.edu
}

\author{
R. Srikant \\ University of Illinois at \\ Urbana-Champaign \\ Urbana, IL, USA \\ rsrikant@illinois.edu
}

\begin{abstract}
Motivated by applications in machine learning and statistics, we study distributed optimization problems over a network of processors, where the goal is to optimize a global objective composed of a sum of local functions. In these problems, due to the large scale of the data sets, the data and computation must be distributed over multiple processors resulting in the need for distributed algorithms. In this paper, we consider a popular distributed gradient-based consensus algorithm, which only requires local computation and communication. An important problem in this area is to analyze the convergence rate of such algorithms in the presence of communication delays that are inevitable in distributed systems. We prove the convergence of the gradient-based consensus algorithm in the presence of uniform, but possibly arbitrarily large, communication delays between the processors. Moreover, we obtain an upper bound on the rate of convergence of the algorithm as a function of the network size, topology, and the inter-processor communication delays.
\end{abstract}

ACM Reference Format:

Thinh T. Doan, Carolyn L. Beck, and R. Srikant. 2018. On the Convergence Rate of Distributed Gradient Methods for Finite-Sum Optimization under Communication Delays: Extended Abstract. In SIGMETRICS'18 Abstracts: ACM SIGMETRICS International Conference on Measurement \& Modeling of Computer Systems Abstracts, June 18-22, 2018, Irvine, CA, USA. ACM, New York, NY, USA, 3 pages. https://doi.org/10.1145/3219617.3219654

\section{INTRODUCTION}

There has been much recent interest in large-scale optimization problems, especially in machine learning and statistics. Due to the explosion in the size of data sets, it is important to be able to solve such problems efficiently. In addition, very often large data sets, on the order of terabytes, cannot be stored or processed on one single processor. As a result, both the data and computation must be distributed over a network of processors, necessitating the development of distributed algorithms. Moreover, the computation

\footnotetext{
${ }^{*}$ The full version of the author's guide is available in [3]

Permission to make digital or hard copies of part or all of this work for personal or classroom use is granted without fee provided that copies are not made or distributed for profit or commercial advantage and that copies bear this notice and the full citation on the first page. Copyrights for third-party components of this work must be honored. For all other uses, contact the owner/author(s).

SIGMETRICS'18 Abstracts, fune 18-22, 2018, Irvine, CA, USA

(C) 2018 Copyright held by the owner/author(s).

ACM ISBN 978-1-4503-5846-0/18/06.

https://doi.org/10.1145/3219617.3219654
}

and communication in these algorithms should be efficient enough so that network latencies do not offset the computational gains.

In this paper, we study distributed gradient methods for optimization problems that are defined over a network of nodes, while explicitly accounting for network delays, one of the most critical issues in distributed systems. In particular, we focus on the convergence in the presence of inter-processor communication delays, which has been identified as an important problem in [4] (see chapter 10). Communication delay, which is one of the most fundamental issues in distributed systems, has been studied in other contexts, such as distributed dual averaging [9]. The analysis in [9] is based on adding fictitious nodes corresponding to the number of time delay steps, thus requiring a modification of the true network topology. As a result, the influence of the delays on the convergence rate for the original network topology is not clear. Convergence under delays are also considered in distributed consensus algorithms $[1,2,6,7,10]$, which are special cases of distributed gradient algorithms. However, these results do not apply to the general distributed algorithms considered here. Our goal in this paper, therefore, is to address this important problem of proving convergence and obtaining convergence rates for distributed gradient algorithms with inter-processor communication delays.

Main Contributions. The main contribution of this paper is to derive the convergence rate of distributed gradient algorithms under uniform communication delays between nodes. In particular, we first show that under some appropriate choice of stepsizes the nodes' estimates asymptotically converge to the solution of the problem, implying that the impact of communication delays is asymptotically negligible. This step allows us to study the rate of convergence of the algorithm, i.e., the convergence occurs at rate $O\left(\frac{n \tau^{3} \ln (t)}{(1-\gamma)^{2} \sqrt{t}}\right)$, where $n$ is the number of processors, $t$ is the time variable, and $\tau$ is the delay constant. In addition, $\gamma$ is a constant in $(0,1)$ that depends on $\sigma_{2}$, the spectral properties of network connectivity of the processors. We note that such an explicit formula for the convergence rate is not available for dual averaging methods. Finally, while we do not analyze dual averaging methods in the presence of delays, we provide simulation results comparing it to distributed gradient methods, which indicate that distributed gradient methods perform significantly better.

\section{PROBLEM FORMULATION}

In this paper, we consider an optimization problem where the objective function is distributed over a network of $n$ nodes. In particular, let $\mathcal{G}=(\mathcal{V}, \mathcal{E})$ be an undirected graph over the vertex set 
$\mathcal{V}=\{1, \ldots n\}$ with the edge set $\mathcal{E}=(\mathcal{V} \times \mathcal{V})$. Associated with each node $i \in \mathcal{V}$ is a convex function $f_{i}: \mathbb{R}^{d} \rightarrow \mathbb{R}$. The goal of the network is to solve the following minimization problem:

$$
\operatorname{minimize} \sum_{i=1}^{n} f_{i}(\mathbf{x}) \text { over } \mathbf{x} \in \mathcal{X},
$$

where $\mathcal{X} \subseteq \mathbb{R}^{d}$ is compact, convex, and known by the nodes. We assume no central coordination between the nodes and since each node knows only a local function $f_{i}$, the nodes are required to cooperatively solve the problem. We are interested in studying distributed consensus-based methods for problem (1) implying that each node $i$ maintains its own parameter estimate $\mathbf{x}_{i} \in \mathbb{R}^{d}$ which is used to estimate the solution of (1). The nodes are only allowed to exchange their estimates with their neighbors through communication constraints imposed by a graph $\mathcal{G}$. The goal is to asymptotically drive the nodes' estimates $\mathbf{x}_{i}$ to $\mathbf{x}^{*}$, a solution of (1).

We conclude this section with additional notation and assumptions which facilitate our development given later. We make the following assumptions throughout the paper.

Assumption 1. The functions $f_{i}$ are convex and differentiable.

Assumption 2. The graph $\mathcal{G}$ is undirected and connected.

Under Assumption 1 and since $\mathcal{X}$ is compact, there exists a point $\mathbf{x}^{*}$ which solves problem (1). However, $\mathbf{x}^{*}$ may not be unique. We will use $\mathcal{X}^{*}$ to denote the set of optimal solutions to problem (1). Moreover, given $\mathbf{x}^{*} \in \mathcal{X}^{*}$ we denote $f^{*}=\sum_{i=1}^{n} f_{i}\left(\mathbf{x}^{*}\right)$. Under Assumption 1 it is obvious that the functions $f_{i}$ are Lipschitz continuous, which we present below as a Proposition for future reference.

Proposition 2.1. Let Assumption 1 hold. Then each function $f_{i}$ is Lipschitz continuous, i.e., there exists a positive constant $C_{i}$ such that

$$
\left|f_{i}(\mathbf{x})-f_{i}(\mathbf{y})\right| \leq C_{i}\|\mathbf{x}-\mathbf{y}\|_{2}, \quad \forall \mathbf{x}, \mathbf{y} \in \mathcal{X}, \forall i \in \mathcal{V} .
$$

\section{DISTRIBUTED GRADIENT METHODS UNDER COMMUNICATION DELAYS}

Discrete-time distributed gradient methods were studied and first analyzed rigorously in [8] for the case of no communication delay; each node $i \in \mathcal{V}$ maintains a variable $x_{i} \in \mathbb{R}$ updated as,

$$
x_{i}(k+1)=\mathcal{P}_{\mathcal{X}}\left[\sum_{j=1}^{n} a_{i j} x_{j}(k)-\alpha(t) f_{i}^{\prime}\left(x_{i}(k)\right)\right],
$$

where $\alpha(t)$ is some sequence of positive stepsizes and $a_{i j}$ is some positive constant. In this paper we focus on the continuous-time version of (3) under the impact of uniform communication delays between nodes. In particular, we assume that at any time $t \geq 0$ node $i$ only receives a delayed value $x_{j}(t-\tau)$ of $x_{j}(t)$ from node $j$, where $\tau$ is a constant representing the time delay of communication between nodes. Each node $i$ (for all $i \in \mathcal{V}$ ) then uses these values to update its estimate as formally stated in (4), where $\mathcal{T}_{\mathcal{X}\left(x_{i}(t)\right)}$ is the tangent cone of $\mathcal{X}$ at $x_{i}(t), \beta$ is some postive constant, and $\alpha(t)$ is a sequence of positive stepsizes. The conditions of $\beta$ and $\alpha(t)$ to guarantee convergence of the algorithm will be explicitly given later. In addition, the initial conditions, $\phi_{i}(t)$, are assumed to be continuous functions of time. Thus, the estimates $x_{i}(t)$ are now functionals since they are functions of $\phi_{i}(t)$. We assume that the delays are uniform across agents, represented by the positive constant $\tau$.
In the sequel, we denote by $\mathrm{A}$ the $n \times n$ weighted adjacency matrix corresponding to $\mathcal{G}$, whose $(i, j)$-th entries are $a_{i j}$. We make the following assumption on $\mathrm{A}$, which is used to guarantee the convergence of the nodes' estimates to a consensus point.

Assumption 3. A is a doubly stochastic matrix, i.e., $\sum_{i=1}^{n} a_{i j}=$ $\sum_{j=1}^{n} a_{i j}=1$. Moreover, $\mathrm{A}$ is assumed to be irreducible and aperiodic. Finally, the weights $a_{i j}>0$ if and only if $(i, j) \in \mathcal{E}$ otherwise $a_{i j}=0$.

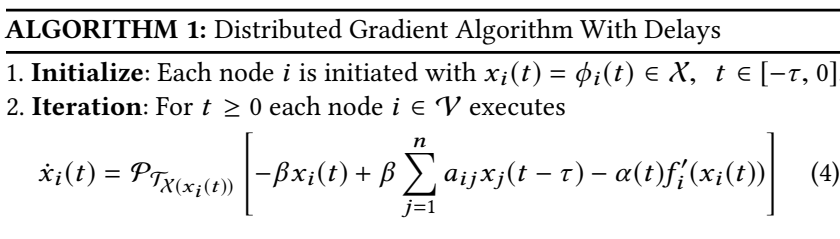

\section{CONVERGENCE RESULTS}

In the following theorem, we show that if each node maintains a variable $z_{i}(t)$ to compute the time-weighted averages of the estimates $x_{i}(t)$ and if the stepsize decays with rate $\alpha(t)=1 / \sqrt{t}$, the algorithm achieves an asymptotic convergence to the optimal value estimated on the variable $z_{i}(t)$ at a rate $O\left(\frac{n \tau^{3} \ln (t)}{(1-\gamma)^{2} \sqrt{t}}\right)$, where $\gamma=\sigma_{2} e^{\beta \tau} \in(0,1)$ and $\beta \in\left(0, \frac{\ln \left(1 / \sigma_{2}\right)}{\tau}\right)$. Here $\sigma_{2}$ represents the algebraic connectivity of the graph $\mathcal{G}$.

Theorem 4.1. Suppose Assumptions 1-3 hold. Let the trajectories of $x_{i}(t)$ be updated by Algorithm 1. Let $\beta \in\left(0, \frac{\ln \left(1 / \sigma_{2}\right)}{\tau}\right)$ and $\gamma=$ $\sigma_{2} e^{\beta \tau} \in(0,1)$. Let $\{\alpha(t)\}$ be a given positive scalar sequence such that $\alpha(t)=1 / \sqrt{t}$ for $t \geq 1$ and $\alpha(t)=1$ for $t \leq 1$. Then $\forall i \in \mathcal{V}$,

$$
F\left(\frac{\int_{0}^{t} \alpha(u) x_{i}(u) d u}{\int_{0}^{t} \alpha(u) d u} \mathbf{1}\right)-f^{*} \leq \frac{2 \Gamma_{0}(t)+n V(\bar{x}(0))}{2 \sqrt{t}-1},
$$

where,

$$
\begin{gathered}
\Gamma_{0}(t) \triangleq \frac{24 C\left(\|\mathbf{x}(0)\|_{2}+2 C\right) e^{\beta \tau / 2}}{\beta^{3}(1-\gamma)^{2}}+\frac{48 C^{2}(1+\tau)}{\beta^{2} \gamma(1-\gamma)}+C^{2} \ln (t) \\
+\frac{48 C^{2} \ln (\gamma t-4 \tau)}{\beta^{2} \gamma(1-\gamma)} .
\end{gathered}
$$

\section{SIMULATIONS}

In this section, we apply the distributed gradient algorithm to study the well-known linear regression problem in statistical machine learning, which is the most popular technique for data fitting. The goal of this problem is to find a linear relationship between a set of variables and some real value outcome. Here, we focus on quadratic loss functions, that is, given a training set $S=\left\{\left(x_{i}, y_{i}\right) \in \mathbb{R}^{d} \times \mathbb{R}\right\}$ for $i=1, \ldots, n$, we want to learn a parameter $w$ that minimizes the following least squares problem,

$$
\min _{w \in X} \sum_{i=1}^{n}\left(x_{i}^{T} w-y_{i}\right)^{2} .
$$

We assume that the data sets are distributedly stored in a network of $n$ processors, i.e., each processor $i$ knows only the pair $\left(x_{i}, y_{i}\right)$.

For the purpose of simulations, we consider the discrete-time version of Algorithm 1, i.e., Eq. (3) with communication delays $\tau$. We simulate for the case when $\mathcal{X}=[-5,5]^{d}$ where $d=10$, i.e., 

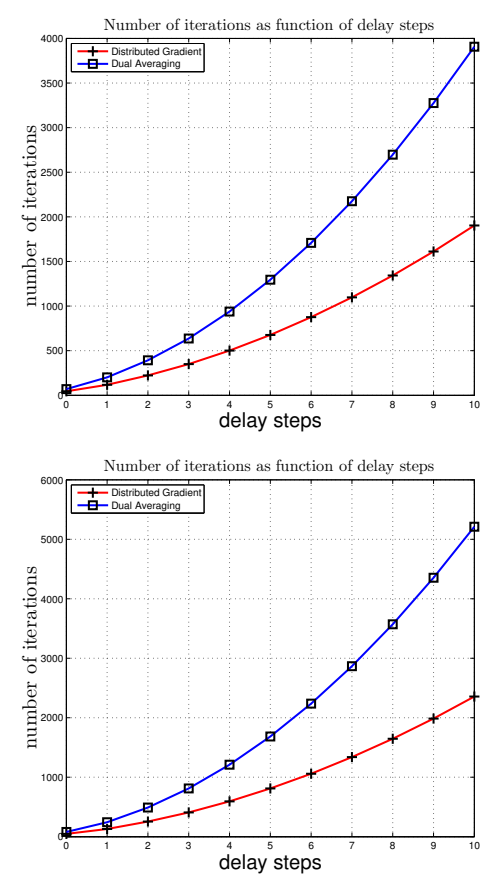

$w, x_{i} \in \mathbb{R}^{10}$. We consider simulated training data sets, i.e., $\left(x_{i}, y_{i}\right)$ are generated randomly with uniform distribution between $[0,1]$. We consider the performance of the distributed gradient algorithm on different sizes of network $\mathcal{G}$. To implement our algorithm, the communication matrix $A$ is chosen as a lazy Metropolis matrix corresponding to $\mathcal{G}$. In all simulations considered herein, we set the stepsize $\alpha(k)=1 / \sqrt{k}$ for $k=1,2, \ldots$ and $\alpha(0)=1$.

We compare the performance of the discretized version of distributed gradient (DG) with distributed dual averaging (DA) [5, 9] for solving problem (7) in the case of constant delays. To study the impact of uniform communication delays on the performance of DG and DA, we implement DG and DA for each network, and terminate them when $\max _{i}\left|F\left(z_{i}(t)\right)-f^{*}\right| \leq 0.2$. We let the delay constant $\tau$ run from 0 to 10 and output the number of iterations as a function on $\tau$. We plot the number of iterations as a function on the number of delay steps. The simulations are shown in Fig. 1.

We first note that the delays do influence the convergence rate of the two algorithms, that is, the greater the delay between nodes the more time the algorithms need to terminate. Second, as shown by the curve for DG the number of iterations seems to increase as a cubic function of the number of delay steps, which agrees with our analysis in Theorem 4.1. Finally, in this example, uniform delays have a bigger impact on the performance of DA, that is, DA requires more iterations to converge than DG under the same number of delay steps.

\section{CONCLUDING REMARKS}

In this paper we have studied a continuous-time distributed gradientbased consensus algorithm for network optimization problems, with the focus on uniform communication delays. We provided an explicit analysis on the rate of convergence of the algorithm as a function of the network size, topology, and communication delays,

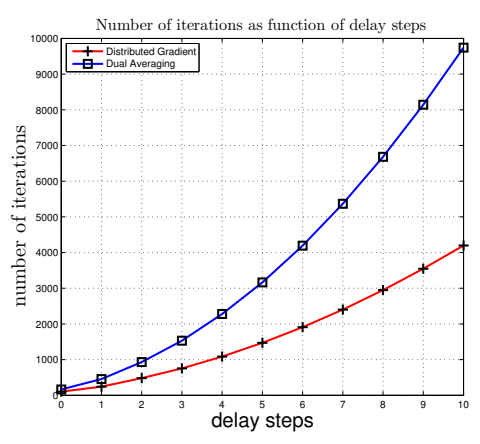

Figure 1: Performance of DG and DA with delays.

specifically the convergence time of the algorithm grows as a cubic function of the delays.

One interesting question left open in this paper is the study of asynchronous distributed gradient algorithms, that is, when communications delays are different at different nodes and perhaps change with time. In this more general case, it would be interesting to investigate whether an upper bound on the time-varying heterogeneous delays can be helpful in obtaining convergence results. In particular, a possible topic of future research would be to determine if one can obtain bounds on the error in the objective function by using an upper bound on the delays, along with our current results.

\section{ACKNOWLEDGMENTS}

The authors would like to thank the anonymous reviewers for their valuable comments and helpful suggestions. The work is supported by Boeing, ARO Grant W911NF-16-1-0259, and the National Science Foundation under Grant NSF CNS 15-44953 and NeTS 1718203.

\section{REFERENCES}

[1] V.D. Blondel, J.M. Hendrickx, A. Olshevsky, and J.N. Tsitsiklis. 2005. Convergence in multiagent coordination, consensus, and flocking. In Proceeding of the foint 44 th Conference on Decision and Control And European Control Conference. 2996-3000.

[2] T. Charalambous, Y. Yuan, T. Yang, W. Pan, C. N. Hadjicostis, and M. Johansson. 2015. Distributed Finite-Time Average Consensus in Digraphs in the Presence of Time Delays. IEEE Transactions on Control of Network Systems 2, 4 (Dec 2015), $370-381$.

[3] T. T. Doan, C. L. Beck, and R. Srikant. 2017. On the Convergence Rate of Distributed Gradient Methods for Finite-Sum Optimization Under Communication Delays. Proc. ACM Meas. Anal. Comput. Syst. 1, 2 (Dec. 2017), 37:1-37:27.

[4] Y.C. Eldar D.P. Palomar. Dec. 2009. Convex Optimization in Signal Processing and Communications (1st ed.). Cambridge University Press.

[5] J.C. Duchi, A. Agarwal, and M.J. Wainwright. 2012. Dual averaging for distributed optimization: Convergence analysis and network scaling. IEEE Transactions on Automatic Control 57, 3 (2012), 592-606.

[6] U. Münz, A. Papachristodoulou, and F. Allgöwer. 2011. Consensus in Multi-Agent Systems With Coupling Delays and Switching Topology. IEEE Trans. Automat. Control 56, 12 (2011), $2976-2982$.

[7] A. Nedić and A. Ozdaglar. 2010. Convergence rate for consensus with delays. Journal of Global Optimization 47, 3 (2010), 437âĂŞ456.

[8] A. Nedić, A. Ozdaglar, and P. A. Parrilo. 2010. Constrained Consensus and Optimization in Multi-Agent Networks. IEEE Trans. Automat. Control 55, 4 (2010), 922-938.

[9] K.I. Tsianos, S. Lawlor, and M.G. Rabbat. 2012. Distributed dual averaging for convex optimization under communication delays. In Proc. of American Control Conference (ACC).

[10] K.I. Tsianos and M.G. Rabbat. 2012. The Impact of Communication Delays on Distributed Consensus Algorithms. arXiv preprint: https://arxiv.org/pdf/1207. 5839.pdf. (2012). 